\title{
酚的三氟甲磺酰基化反应
}

\author{
白小东 $a$ 付志宏* ${ }^{*}$ 曹育才 ${ }^{c}$ 林锦鸿 $* b$ \\ $\left({ }^{a}\right.$ 西南石油大学新能源与材料学院 成都 610500) \\ ( ${ }^{b}$ 中国科学院上海有机化学研究所 有机氟化学重点实验室 中国科学院大学 上海 200032) \\ ${ }^{c}$ 上海化工研究院有限公司 聚烯烃催化技术与高性能材料国家重点实验室 \\ 上海市聚烯烃催化技术重点实验室 上海 200062)
}

\begin{abstract}
摘要 三氟甲磺酸芳基酯在有机合成和药物化学中有重要应用, 因而如何实现高效制备吸引了不少关注. 三氟甲磺酰 基吡啶盐 $\left(\mathrm{C}_{5} \mathrm{H}_{5} \mathrm{~N}^{+} \mathrm{SO}_{2} \mathrm{CF}_{3} \cdot \mathrm{CF}_{3} \mathrm{SO}_{3}{ }^{-}\right)$可作为温和的三氟甲磺酰基化试剂，与酚类化合物反应得到三氟甲磺酸芳基酯. 产 物的纯化只需简单洗涤，不需要繁琐的柱层析操作．除了芳基酯，烯基酯也能由该吡啶盐为试剂合成得到. 吡啶盐的 制备和分离容易，干燥环境中稳定存在，使用起来方便.
\end{abstract}

关键词 三氟甲磺酸芳基酯; 三氟甲磺酰基化; 酚; 三氟甲磺酰基吡啶盐; 氟

\section{Trifluoromethanesulfonylation of Phenols}

\author{
Bai, Xiaodong ${ }^{a}$ \\ $\mathrm{Fu}$, Zhihong ${ }^{*, a}$ \\ Cao, Yucai ${ }^{\mathrm{C}}$ \\ Lin, Jinhong*,b
}

( ${ }^{a}$ School of New Energy and Materials, Southwest Petroleum University, Chengdu 610500)

( ${ }^{b}$ Key Laboratory of Organofluorine Chemistry, Shanghai Institute of Organic Chemistry, University of

Chinese Academy of Sciences, Chinese Academy of Sciences, Shanghai 200032)

( ${ }^{c}$ State Key Laboratory of Polyolefins and Catalysis, Shanghai Key Laboratory of Catalysis Technology for Polyolefins, Shanghai Research Institute of Chemical Industry Co. Ltd., Shanghai 200062)

\begin{abstract}
As aryl triflates are widely used in organic synthesis and medicinal chemistry, significant efforts have been directed towards the development of efficient methods for their synthesis. It was found that trifluoromethanesulfonyl pyridinium salt $\left(\mathrm{C}_{5} \mathrm{H}_{5} \mathrm{~N}^{+} \mathrm{SO}_{2} \mathrm{CF}_{3} \cdot \mathrm{CF}_{3} \mathrm{SO}_{3}{ }^{-}\right)$was able to act as a mild trifluoromethanesulfonylation reagent to convert phenols into aryl triflates. All aryl triflate products could be purified simply by washing, and tedious chromatography operations were avoided. Besides aryl triflates, vinyl triflates could also be synthesized by using this pyridinium salt as reagent. The pyridinium salt could be easily prepared and purified, is stable under dry atmosphere, and thus may become an easy-to-handle reagent.

Keywords aryl triflates; trifluoromethanesulfonylation; phenols; trifluoromethanesulfonyl pyridinium salt; fluorine
\end{abstract}

\section{Introduction}

Aryl triflates have found widespread applications in organic synthesis ${ }^{[1]}$ and medicinal chemistry ${ }^{[2]}$. They have served as versatile coupling partners in transition metal-catalyzed cross-coupling reactions. ${ }^{[1]}$ Aryl triflates bearing a trimethylsilyl group at the ortho position have proved to be convenient aryne precursors. ${ }^{[3]}$ Furthermore, the trifluoromethyl group could increase the lipophilicity of organic compounds, and thus aryl triflate moiety can be present as an important structural motif in medicinal chemistry. ${ }^{[2]}$ Therefore, significant efforts have been directed towards the development of efficient methods for the synthesis of aryl triflates. Although $\mathrm{C}-\mathrm{H}$ triflation is a step-economy strategy, it suffers from a limited substrate scope or the need for the installation of a directing group into substrates (Scheme 1, a). ${ }^{[4]}$ The Sandmeyer triflation may deliver products which are difficult to obtain by other routes, but the arenediazonium substrates are explosive and high triflation temperatures may be required (Scheme 1,

\footnotetext{
* Corresponding authors. E-mail: 1160757400@qq.com; jlin@sioc.ac.cn

Received November 22, 2019; revised December 27, 2019; published online January 3, 2020.

Project supported by the National Natural Science Foundation of China (Nos. 21421002, 21672242, 21971252, 51104122), the Key Research Program of Frontier Sciences, Chinese Academy of Sciences (CAS) (No. QYZDJSSW-SLH049), the Youth Innovation Promotion Association CAS (No. 2019256), the Fujian Institute of Innovation, CAS (No. FJCXY18040102), and the Shanghai Research Institute of Chemical Industry Co., LTD. (No. SKL-LCTP-201802). 国家自然科学基金(Nos. 21421002, 21672242, 21971252, 51104122)、中国科学院前沿科学重点研究计划(No. QYZDJSSW-SLH049)、中国科学院青年 创新促进会(No. 2019256)、中国科学院福建创新研究院(No. FJCXY18040102)和上海化工研究院有限公司(No. SKL-LCTP-201802)资助项目.
} 
b). ${ }^{[5]}$ Trifluoromethanesulfonylation of phenols, the most commonly used protocol, is quite straightforward and attractive. ${ }^{[6]}$

Various trifluoromethanesulfonylation reagents, such as $\mathrm{TfCl}\left(\mathrm{Tf}=\mathrm{CF}_{3} \mathrm{SO}_{2}\right),{ }^{[7]} \mathrm{Tf}_{2} \mathrm{O},{ }^{[8]}$ Im-Tf $(\mathrm{Im}=$ imidazole $),{ }^{[9]}$ PhNTf $_{2},{ }^{[10]}$ and ArOTf, ${ }^{[11]}$ have been developed for the synthesis of aryl triflates from phenols (Scheme 1, c). The good reactivity of $\mathrm{PhNTf}_{2}$ has stimulated the development of polymer-supported reagents, polymer- $\mathrm{C}_{6} \mathrm{H}_{4} \mathrm{NTf}_{2}$, whose byproduct, polymer- $\mathrm{C}_{6} \mathrm{H}_{4} \mathrm{NHTf}$, generated in the trifluoromethane- sulfonylation reactions, could be recovered and reused to generate polymer- $\mathrm{C}_{6} \mathrm{H}_{4} \mathrm{NTf}_{2}{ }^{\left[{ }^{12]}\right.}$ Although these reagents are quite efficient, some drawbacks still remain. For instance, $\mathrm{TfCl}$ and $\mathrm{Tf}_{2} \mathrm{O}$ are highly volatile and may lead to inconvenient operations. Tedious procedures are required for the synthesis and the reuse of the polymer-supported reagents. The desired products usually have to be isolated by flash column chromatography. Therefore, the development of efficient trifluoromethanesulfonylation reagents is desirable.

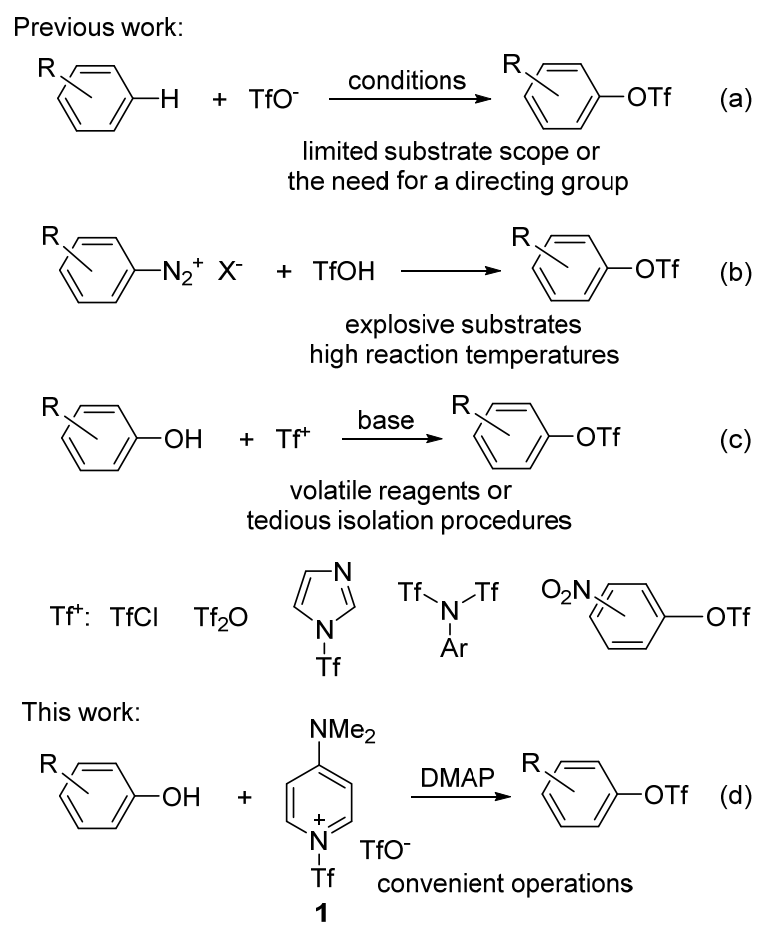

Scheme 1 Synthesis of aryl triflates

Although the trifluoromethanesulfonyl pyridinium salt, $\mathbf{1}$, is a known compound, ${ }^{[13]}$ it has never been used as trifluoromethanesulfonylation reagent. We found that this compound could smoothly convert phenols into aryl triflates under mild conditions (Scheme 1, d). More importantly, the pure triflate products could be isolated simply by washing. The preliminary results are described herein.

\section{Results and discussion}

Pyridinium salt $\mathbf{1}$ could be readily produced from the reaction of pyridine with trifluoromethanesulfonic anhydride, and the pure product could be obtained via a simple washing procedure (please see experimental section). It is a solid and stable under dry atmosphere. Our initial attempts at trifluoromethanesulfonylation of substrate $\mathbf{2 a}$ with salt $\mathbf{1}$ revealed that almost a quantitative yield could be obtained by using organic or inorganic base (Table 1, Entries $1 \sim 6$ ). Decreasing the loading of DMAP did not lower the yield (Entries 7 9). Interestingly, the desired product was also obtained in $99 \%{ }^{19} \mathrm{~F}$ NMR yield when using 0.1 equiv. of other base such as $\mathrm{Et}_{3} \mathrm{~N}$ or DBU. 0.1 equiv. of $\mathrm{NaHCO}_{3}$ gave the desired product only in $33 \%{ }^{19} \mathrm{~F}$ NMR yield. However, no desired product was generated and salt 1 remained intact if no base was used (Entry 10). The reaction proceeded smoothly in various reaction solvents (Entry 9 and Entries 11 13). The yield was not decreased by shortening the reaction time (Entries 14).

Table 1 Optimization of reaction conditions ${ }^{a}$

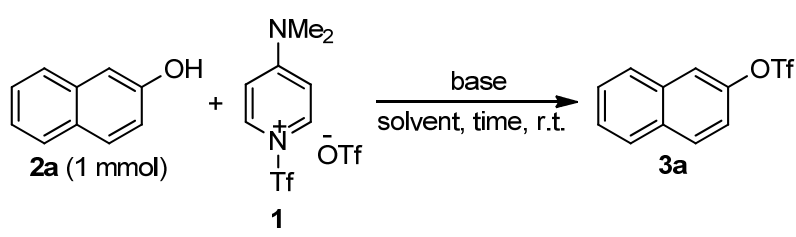

\begin{tabular}{|c|c|c|c|c|}
\hline Entry & Base (mmol) & Solvent & Time/h & Yield $^{b} / \%$ \\
\hline 1 & $\mathrm{Et}_{3} \mathrm{~N}(1.5)$ & DCM & 3 & 99 \\
\hline 2 & DBU (1.5) & DCM & 3 & 99 \\
\hline 3 & Pyridine (1.5) & DCM & 3 & 99 \\
\hline 4 & DMAP (1.5) & DCM & 3 & 99 \\
\hline 5 & $\mathrm{NaHCO}_{3}(1.5)$ & DCM & 3 & 99 \\
\hline 6 & $\mathrm{Na}_{2} \mathrm{CO}_{3}(1.5)$ & DCM & 3 & 99 \\
\hline 7 & DMAP (1.0) & DCM & 3 & 99 \\
\hline 8 & DMAP (0.5) & DCM & 3 & 99 \\
\hline 9 & DMAP $(0.1)$ & DCM & 3 & 99 \\
\hline 10 & - & DCM & 3 & ND \\
\hline 11 & DMAP $(0.1)$ & EA & 3 & 99 \\
\hline 12 & DMAP $(0.1)$ & n-Hexane & 3 & 99 \\
\hline 13 & DMAP (0.1) & Acetone & 3 & 99 \\
\hline 14 & DMAP (0.1) & DCM & 0.5 & 99 \\
\hline
\end{tabular}

${ }^{a}$ Reaction conditions: Substrate 2a $(1 \mathrm{mmol})$, reagent $1(1.9 \mathrm{mmol})$, base, and solvent $(5 \mathrm{~mL})$ at room temperature under air atmosphere; DBU $=1,8-\mathrm{Di}$ azabicyclo[5.4.0] undec-7-ene; DMAP =4-dimethylaminopyridine; $\mathrm{EA}=$ ethyl acetate; $\mathrm{ND}=$ not detected; ${ }^{b}$ The yields were determined by ${ }^{19} \mathrm{~F}$ NMR spectroscopy by using $\mathrm{PhOCF}_{3}$ as an internal standard.

With the optimal reaction conditions in hand (Table 1, Entry 14), we then investigated the substrate scope of the trifluoromethanesulfonylation of phenols with salt 1. As shown in Scheme 2, various electron-rich, electron-neutral and electron-deficient phenols were all converted smoothly into the desired products in high yields. Estrone, a female sex hormone, could also be transformed into its corresponding triflate under these conditions (3n). It is worth noting that all of these products were purified simply by washing without the use of chromatography. Increasing the reaction scale to $10 \mathrm{mmol}$, product $\mathbf{3 a}$ was still afforded in 
high isolated yield (89\%), further demonstrating the synthetic utility of this protocol.

Table 2 Trifluoromethanesulfonylation of phenols ${ }^{a}$
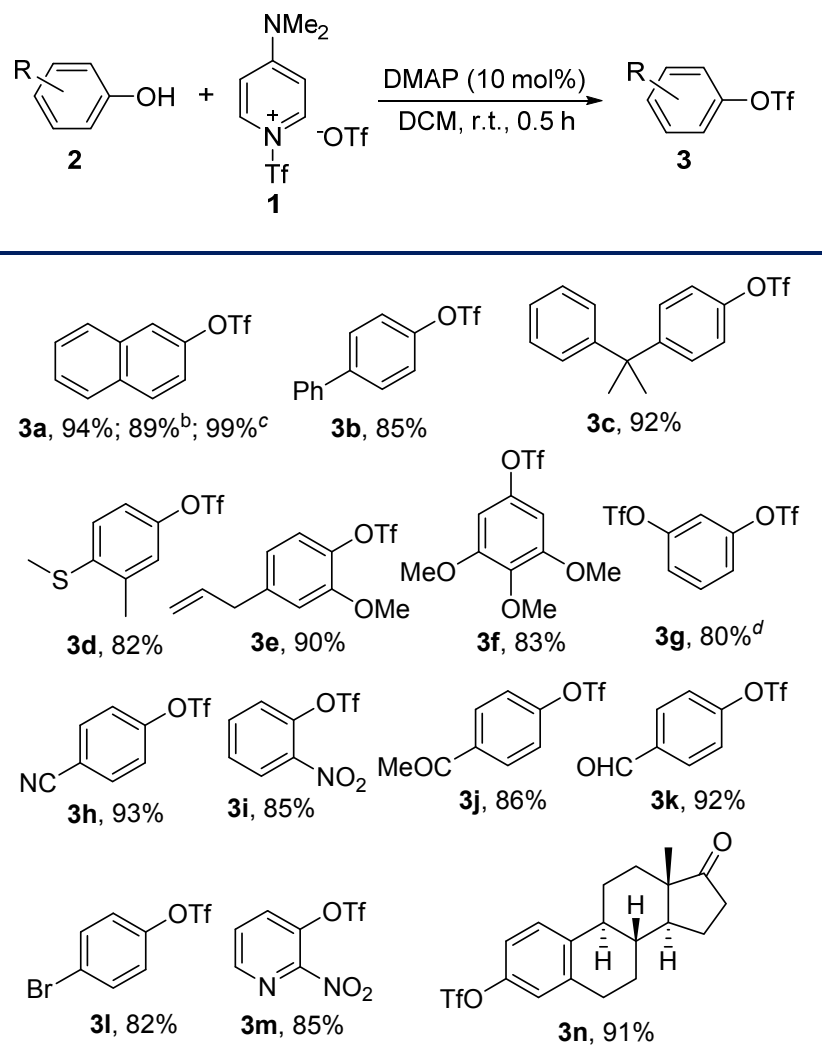

${ }^{a}$ Reaction conditions: substrate $2(1 \mathrm{mmol})$, salt 1 (1.9 mmol), DMAP $(0.1$ $\mathrm{mmol})$, DCM $(5 \mathrm{~mL})$ at room temperature for $0.5 \mathrm{~h}$. Isolated yields. All products were purified simply by washing. ${ }^{b}$ A 10 mmol-scale reaction gave product 3a in $89 \%$ yield; ${ }^{c}$ A $99 \%{ }^{19} \mathrm{~F}$ NMR yield was obtained by using 1.1 equiv. of salt $1 ;{ }^{d}$ Resorcinol was used as the substrate and $3.8 \mathrm{mmol}$ of salt $\mathbf{1}$ was used.

Vinyl triflates are also valuable coupling partners in transition metal-catalyzed cross-coupling reactions. ${ }^{[6]}$ $\beta$-Ketoesters could also be transformed into vinyl triflates by using salt 1 as a trifluoromethanesulfonylation reagent (Scheme 2). The one-pot two-step reaction gave the desired products in high yields.<smiles>CCOC(=O)C1CCCC1=O</smiles>
4

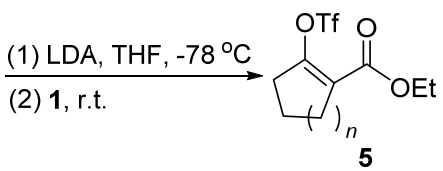

5 a, $n=1,86 \%$ 5b, $n=2,83 \%$
(1) 4 (1 mmol), LDA $(1 \mathrm{mmol})$ in THF $(10 \mathrm{ml})$ at $-78^{\circ} \mathrm{C}$ for $1 \mathrm{~h}$

(2) $1(1.9 \mathrm{mmol})$ at room temperature for $12 \mathrm{~h}$

Scheme 2 Synthesis of vinyl triflates

\section{Conclusions}

In summary, the trifluoromethanesulfonylation of phenols with pyridinium salt $\mathbf{1}$ under mild conditions to give the desired aryl triflates in high yields have been described. All triflate products were purified simply by washing without the use of chromatography. Vinyl triflates were also synthesized from $\beta$-ketoesters by using salt $\mathbf{1}$ as reagent. The trifluoromethanesulfonylation protocol is quite attractive due to the easy accessibility of the reagent and the convenient operations for product purification.

\section{Experimental section}

\subsection{General information}

${ }^{1} \mathrm{H}$ NMR and ${ }^{19} \mathrm{~F}$ NMR spectra were detected on a 500 , 400 or $300 \mathrm{MHz}$ NMR spectrometer. Mass spectra were obtained on GC-MS. Unless otherwise noted, all reagents were obtained commercially and used without further purification.

\subsubsection{Procedure for the synthesis of pyridinium salt 1}

Into the solution of DMAP ( $0.1 \mathrm{~mol}, 12.2 \mathrm{~g}, 1.0$ equiv.) in DCM $(40 \mathrm{~mL})$ was added $\mathrm{Tf}_{2} \mathrm{O}(0.15 \mathrm{~mol}, 42.3 \mathrm{~g}, 1.5$ equiv) slowly at $0{ }^{\circ} \mathrm{C}$. The resulting mixture was warmed to room temperature and stirred for $1 \mathrm{~h}$. The precipitate was washed with DCM, and the solid was dried under vacuum to afford 4-dimethylamino-1-(trifluoromethyl)sulfonylpyridin-1-ium trifluoromethanesulfonate (1) ${ }^{[13]}$ (34.3 g, 85\% yield). ${ }^{1} \mathrm{H}$ NMR (400 MHz, $\left.\mathrm{CD}_{3} \mathrm{CN}\right) \delta: 8.28$ (d, $J=8.2 \mathrm{~Hz}, 2 \mathrm{H}), 7.13(\mathrm{~d}, J=8.3 \mathrm{~Hz}, 2 \mathrm{H}), 3.41(\mathrm{~s}, 6 \mathrm{H})$; ${ }^{19} \mathrm{~F}$ NMR $\left(376 \mathrm{MHz}, \mathrm{CD}_{3} \mathrm{CN}\right) \delta:-74.59(\mathrm{~s}, 3 \mathrm{~F}),-79.36$ (s, 3F).

4.2.2 Typical procedure for the trifluoromethanesulfonylation of phenols

Into a $15 \mathrm{~mL}$ Schlenk tube were added substrate 2 (1.0 mmol), pyridinium salt 1 (1.9 mmol, $756.0 \mathrm{mg})$, DMAP $(0.1 \mathrm{mmol}, 12.2 \mathrm{mg})$ and DCM $(5 \mathrm{~mL})$. The tube was sealed and the resulting mixture was stirred at room temperature for $0.5 \mathrm{~h}$. The reaction was quenched by water. The organic phase was washed with water $(10 \mathrm{~mL} \times 3)$, and the water phase was further extracted with DCM. The combined organic phase was concentrated and the solvent was removed to afford the pure product 3 .

Naphthalen-2-yl trifluoromethanesulfonate (3a): ${ }^{[14]} 94 \%$ yield. ${ }^{1} \mathrm{H}$ NMR $\left(400 \mathrm{MHz}, \mathrm{CDCl}_{3}\right) \delta: 7.93 \sim 7.81(\mathrm{~m}, 3 \mathrm{H})$, $7.76(\mathrm{~d}, J=2.2 \mathrm{~Hz}, 1 \mathrm{H}), 7.62 \sim 7.51(\mathrm{~m}, 2 \mathrm{H}), 7.38(\mathrm{dd}$, $J=9.0,2.4 \mathrm{~Hz}, 1 \mathrm{H}) ;{ }^{19} \mathrm{~F} \mathrm{NMR}\left(376 \mathrm{MHz}, \mathrm{CDCl}_{3}\right) \delta$ : -72.88 (s, 3F); GC-MS m/z: 276.0 .

(1,1'-Biphenyl)-4-yl trifluoromethanesulfonate $(\mathbf{3 b}):^{[14]}$ $85 \%$ yield. ${ }^{1} \mathrm{H}$ NMR $\left(400 \mathrm{MHz}, \mathrm{CDCl}_{3}\right) \delta: 7.65(\mathrm{~d}, J=8.8$ $\mathrm{Hz}, 2 \mathrm{H}), 7.58(\mathrm{~d}, J=7.1 \mathrm{~Hz}, 2 \mathrm{H}), 7.53 \sim 7.40(\mathrm{~m}, 3 \mathrm{H})$, $7.37(\mathrm{~d}, J=8.8 \mathrm{~Hz}, 2 \mathrm{H}) ;{ }^{19} \mathrm{~F}$ NMR $\left(376 \mathrm{MHz}, \mathrm{CDCl}_{3}\right) \delta$ : -72.88 (s, 3F); GC-MS m/z: 302.0 .

4-(2-Phenylpropan-2-yl)phenyl trifluoromethanesulfonate $(\mathbf{3 c})::^{[15]} 92 \%$ yield. ${ }^{1} \mathrm{H}$ NMR $\left(400 \mathrm{MHz}, \mathrm{CDCl}_{3}\right) \delta$ : $7.38 \sim 7.34(\mathrm{~m}, 4 \mathrm{H}), 7.32 \sim 7.26(\mathrm{~m}, 3 \mathrm{H}), 7.25 \sim 7.20(\mathrm{~m}$, $2 \mathrm{H}), 1.76(\mathrm{~s}, 6 \mathrm{H}) ;{ }^{19} \mathrm{~F}$ NMR $\left(376 \mathrm{MHz}, \mathrm{CDCl}_{3}\right) \delta:-73.01$ (s, 3F); GC-MS m/z: 344.1.

3-Methyl-4-(methylthio)phenyl trifluoromethanesulfo- 
nate $(\mathbf{3 d})::^{[14]} 82 \%$ yield. ${ }^{1} \mathrm{H}$ NMR $\left(400 \mathrm{MHz}, \mathrm{CDCl}_{3}\right) \delta$ : $7.13(\mathrm{~d}, J=8.6 \mathrm{~Hz}, 1 \mathrm{H}), 7.11 \sim 7.03(\mathrm{~m}, 2 \mathrm{H}), 2.45(\mathrm{~s}, 3 \mathrm{H})$, $2.32(\mathrm{~s}, 3 \mathrm{H}) ;{ }^{19} \mathrm{~F}$ NMR $\left(376 \mathrm{MHz}, \mathrm{CDCl}_{3}\right) \delta:-73.06(\mathrm{~s}$, $3 \mathrm{~F}$ ); GC-MS $m / z$ : found 286.0 .

4-Allyl-2-methoxyphenyl trifluoromethanesulfonate (3e) ${ }^{[16]} 90 \%$ yield. ${ }^{1} \mathrm{H}$ NMR $\left(400 \mathrm{MHz}, \mathrm{CDCl}_{3}\right) \delta: 7.12(\mathrm{~d}$, $J=8.3 \mathrm{~Hz}, 1 \mathrm{H}), 6.86(\mathrm{~d}, J=1.5 \mathrm{~Hz}, 1 \mathrm{H}), 6.79(\mathrm{dd}, J=8.3$, $1.4 \mathrm{~Hz}, 1 \mathrm{H}), 6.02 \sim 5.87(\mathrm{~m}, 1 \mathrm{H}), 5.18 \sim 5.07(\mathrm{~m}, 2 \mathrm{H}), 3.88$ (s, 3H), $3.39(\mathrm{~d}, J=6.7 \mathrm{~Hz}, 2 \mathrm{H}) ;{ }^{19} \mathrm{~F}$ NMR $(376 \mathrm{MHz}$, $\left.\mathrm{CDCl}_{3}\right) \delta$ : $-74.06(\mathrm{~s}, 3 \mathrm{~F})$; GC-MS $\mathrm{m} / z$ : found 296.0.

3,4,5-Trimethoxyphenyl trifluoromethanesulfonate (3f): ${ }^{[14]} 83 \%$ yield. ${ }^{1} \mathrm{H}$ NMR $\left(400 \mathrm{MHz}, \mathrm{CDCl}_{3}\right) \delta: 6.44$ (s, 2H), $3.79(\mathrm{~s}, 6 \mathrm{H}), 3.77(\mathrm{~s}, 3 \mathrm{H}) ;{ }^{19} \mathrm{~F}$ NMR $(376 \mathrm{MHz}$, $\left.\mathrm{CDCl}_{3}\right) \delta:-73.06$ (s, 3F); GC-MS $m / z: 316.0$.

1,3-Phenylene bis(trifluoromethanesulfonate) (3g) ${ }^{[17]}$ $80 \%$ yield. ${ }^{1} \mathrm{H}$ NMR $\left(400 \mathrm{MHz}, \mathrm{CDCl}_{3}\right) \delta: 7.56$ (t, $J=8.4$ $\mathrm{Hz}, 1 \mathrm{H}), 7.35$ (dd, $J=8.4,2.3 \mathrm{~Hz}, 2 \mathrm{H}), 7.26(\mathrm{t}, J=2.2 \mathrm{~Hz}$, $1 \mathrm{H}) ;{ }^{19} \mathrm{~F}$ NMR (376 MHz, $\left.\mathrm{CDCl}_{3}\right) \delta:-72.88(\mathrm{~s}, 6 \mathrm{~F})$; GC-MS $m / z: 373.9$.

4-Cyanophenyl trifluoromethanesulfonate (3h): ${ }^{[10 b]} 93 \%$ yield. ${ }^{1} \mathrm{H}$ NMR $\left(400 \mathrm{MHz}, \mathrm{CDCl}_{3}\right) \delta: 7.77(\mathrm{~d}, J=8.9 \mathrm{~Hz}$, $2 \mathrm{H}), 7.40(\mathrm{~d}, J=8.9 \mathrm{~Hz}, 2 \mathrm{H}) ;{ }^{19} \mathrm{~F}$ NMR $\left(376 \mathrm{MHz}, \mathrm{CDCl}_{3}\right)$ $\delta$ : -72.79 (s, 3F); GC-MS m/z: 251.0.

2-Nitrophenyl trifluoromethanesulfonate $(\mathbf{3 i}){ }^{[18]} 85 \%$ yield. ${ }^{1} \mathrm{H}$ NMR $\left(400 \mathrm{MHz}, \mathrm{CDCl}_{3}\right) \delta: 8.14$ (dd, $J=8.2,1.6$ $\mathrm{Hz}, 1 \mathrm{H}), 7.76(\mathrm{td}, J=8.3,1.7 \mathrm{~Hz}, 1 \mathrm{H}), 7.59(\mathrm{td}, J=8.2,1.2$ $\mathrm{Hz}, 1 \mathrm{H}), 7.45(\mathrm{~d}, J=8.3 \mathrm{~Hz}, 1 \mathrm{H}) ;{ }^{19} \mathrm{~F}$ NMR $(376 \mathrm{MHz}$, $\left.\mathrm{CDCl}_{3}\right) \delta:-73.29$ (s, 3F); GC-MS m/z: 270.9.

4-Acetylphenyl trifluoromethanesulfonate $(\mathbf{3 j}):^{[14]} 86 \%$ yield. ${ }^{1} \mathrm{H}$ NMR $\left(400 \mathrm{MHz}, \mathrm{CDCl}_{3}\right) \delta: 8.00(\mathrm{~d}, J=8.9 \mathrm{~Hz}$, $2 \mathrm{H}), 7.32(\mathrm{~d}, J=8.8 \mathrm{~Hz}, 2 \mathrm{H}), 2.56(\mathrm{~s}, 3 \mathrm{H}) ;{ }^{19} \mathrm{~F}$ NMR $(376$ $\mathrm{MHz}, \mathrm{CDCl}_{3}$ ) $\delta:-73.02$ (s, 3F); GC-MS m/z: 268.0.

4-Formylphenyl trifluoromethanesulfonate (3k): ${ }^{[19]} 92 \%$ yield. ${ }^{1} \mathrm{H}$ NMR (400 MHz, $\left.\mathrm{CDCl}_{3}\right) \delta: 10.00(\mathrm{~s}, 1 \mathrm{H}), 7.96$ $(\mathrm{d}, J=8.8 \mathrm{~Hz}, 2 \mathrm{H}), 7.42(\mathrm{~d}, J=8.7 \mathrm{~Hz}, 2 \mathrm{H}) ;{ }^{19} \mathrm{~F}$ NMR $\left(376 \mathrm{MHz}, \mathrm{CDCl}_{3}\right) \delta:-72.94$ (s, 3F); GC-MS m/z: 254.0 .

4-Bromophenyl trifluoromethanesulfonate $(\mathbf{3 I})^{[19]}: 82 \%$ yield. ${ }^{1} \mathrm{H}$ NMR $\left(400 \mathrm{MHz}, \mathrm{CDCl}_{3}\right) \delta: 7.62 \sim 7.52(\mathrm{~m}, 2 \mathrm{H})$, $7.19 \sim 7.12(\mathrm{~m}, 2 \mathrm{H}) ;{ }^{19} \mathrm{~F}$ NMR $\left(376 \mathrm{MHz}, \mathrm{CDCl}_{3}\right) \delta$ : -72.77 (3F); GC-MS m/z: 303.9.

2-Nitropyridin-3-yl trifluoromethanesulfonate $(\mathbf{3 m}):^{[20]}$ $85 \%$ yield. ${ }^{1} \mathrm{H}$ NMR $\left(400 \mathrm{MHz}, \mathrm{CDCl}_{3}\right) \delta: 8.58$ (dd, $J=$ $4.5,1.3 \mathrm{~Hz}, 1 \mathrm{H}), 7.95(\mathrm{dd}, J=8.3,1.1 \mathrm{~Hz}, 1 \mathrm{H}), 7.82(\mathrm{dd}$, $J=8.3,4.5 \mathrm{~Hz}, 1 \mathrm{H}) ;{ }^{19} \mathrm{~F}$ NMR $\left(376 \mathrm{MHz}, \mathrm{CDCl}_{3}\right) \delta$ : -73.08 (s, 3F); GC-MS m/z: 271.9 .

$(8 R, 9 S, 13 S, 14 S)$-13-Methyl-17-oxo-7,8,9,11,12,13,14, 15,16,17-decahydro- $6 H$-cyclopenta[ $a$ ] phenanthren-3-yl trifluoromethanesulfonate (3n): ${ }^{[14]} 91 \%$ yield. ${ }^{1} \mathrm{H}$ NMR $\left(400 \mathrm{MHz}, \mathrm{CDCl}_{3}\right) \delta: 7.29(\mathrm{~d}, J=8.6 \mathrm{~Hz}, 1 \mathrm{H}), 7.02 \sim 6.89$ (m, $2 \mathrm{H}), 2.91 \sim 2.88(\mathrm{~m}, 2 \mathrm{H}), 2.45(\mathrm{dd}, J=18.5,8.7 \mathrm{~Hz}$, $1 \mathrm{H}), 2.39 \sim 2.30(\mathrm{~m}, 1 \mathrm{H}), 2.29 \sim 2.19(\mathrm{~m}, 1 \mathrm{H}), 2.16 \sim 1.96$ $(\mathrm{m}, 3 \mathrm{H}), 1.95 \sim 1.88(\mathrm{~m}, 1 \mathrm{H}), 1.68 \sim 1.34(\mathrm{~m}, 6 \mathrm{H}), 0.86(\mathrm{~s}$, $3 \mathrm{H}) ;{ }^{19} \mathrm{~F}$ NMR $\left(376 \mathrm{MHz}, \mathrm{CDCl}_{3}\right) \delta$ : -73.12 (s, 3F); GC-MS $m / z: 402.1$.

4.2.3 Procedure for the $10 \mathrm{mmol}$-scale reaction

Into a mixture of $2 \mathrm{a}(10.0 \mathrm{mmol}, 1.44 \mathrm{~g})$, pyridinium salt 1 (19 mmol, $7.56 \mathrm{~g})$, DMAP ( $1 \mathrm{mmol}, 122 \mathrm{mg})$ and DCM $(40 \mathrm{~mL})$. The resulting mixture was stirred at room temperature for $0.5 \mathrm{~h}$. The reaction was quenched by water. The organic phase was washed with water $(20 \mathrm{~mL} \times 3)$, and the water phase was further extracted with DCM. The combined organic phase was concentrated to remove the solvent to afford the pure product 3a (2.45 g, 89\%).

4.2.4 Typical procedure for the synthesis of vinyl triflates

Into the solution of substrate $4(1.0 \mathrm{mmol})$ in dry THF $(10 \mathrm{~mL})$ was added LDA $(0.5 \mathrm{~mL}, 2 \mathrm{~mol} / \mathrm{L})$ at $-78{ }^{\circ} \mathrm{C}$. The mixture was stirred at the same temperature for $1 \mathrm{~h}$. Pyridinium salt $1(1.9 \mathrm{mmol}, 756.0 \mathrm{mg})$ was added. The reaction mixture was warmed to room temperature and stirred at room temperature for $12 \mathrm{~h}$. Products 5 were isolated by flash column chromatography.

2-(((Trifluoromethyl)sulfonyl)oxy)cyclopent-1-ene-1carboxylate (5a): ${ }^{[21]} 86 \%$ yield. ${ }^{1} \mathrm{H}$ NMR $(400 \mathrm{MHz}$, $\left.\mathrm{CDCl}_{3}\right) \delta: 4.21(\mathrm{q}, J=7.2 \mathrm{~Hz}, 2 \mathrm{H}), 2.72 \sim 2.63(\mathrm{~m}, 4 \mathrm{H})$, $2.00 \sim 1.93(\mathrm{~m}, 2 \mathrm{H}), 1.27(\mathrm{t}, J=7.1 \mathrm{~Hz}, 3 \mathrm{H}) ;{ }^{19} \mathrm{~F}$ NMR $\left(376 \mathrm{MHz}, \mathrm{CDCl}_{3}\right) \delta:-74.79$ (s, 3F); GC-MS m/z: 288.0.

2-(((Trifluoromethyl)sulfonyl)oxy)cyclohex-1-ene-1carboxylate (5b): ${ }^{[22]} 83 \%$ yield. ${ }^{1} \mathrm{H}$ NMR $(400 \mathrm{MHz}$, $\left.\mathrm{CDCl}_{3}\right) \delta: 4.23(\mathrm{q}, J=7.1 \mathrm{~Hz}, 2 \mathrm{H}), 2.50 \sim 2.40(\mathrm{~m}, 2 \mathrm{H})$, $2.40 \sim 2.31(\mathrm{~m}, 2 \mathrm{H}), 1.78 \sim 1.72(\mathrm{~m}, 2 \mathrm{H}), 1.66 \sim 1.60(\mathrm{~m}$, $2 \mathrm{H}), 1.29(\mathrm{t}, J=7.5 \mathrm{~Hz}, 3 \mathrm{H}) ;{ }^{19} \mathrm{~F}$ NMR $\left(376 \mathrm{MHz}, \mathrm{CDCl}_{3}\right)$ $\delta:-74.91$ (s, 3F); GC-MS m/z: 302.0.

Supporting Information ${ }^{1} \mathrm{H}$ NMR and ${ }^{19} \mathrm{~F}$ NMR spectra of salt $\mathbf{1}$, products $\mathbf{3 a} \sim \mathbf{3 n}$ and $\mathbf{5 a} \sim \mathbf{5 b}$. The Supporting Information is available free of charge via the Internet at http://sioc-journal.cn/.

\section{References}

[1] (a) Miyaura, N.; Suzuki, A. Chem. Rev. 1995, 95, 2457. (b) Hartwig, J. F. Angew. Chem., Int. Ed. 1998, 37, 2046. (c) Zhang, M.; Chen, B.; Ge, C.; Liu, R.; Gao, J.; Jia, Y. Chin. J. Org. Chem. 2016, 36, 1636 (in Chinese).

(张鸣頔, 陈斌, 葛晨, 刘人荣, 高建荣, 贾义霞, 有机化学, 2016, 36, 1636.)

[2] (a) Moriconi, A.; Bigogno, C.; Bianchini, G.; Caligiuri, A.; Resconi, A.; Dondio, M. G.; D’Anniballe, G.; Allegretti, M. ACS Med. Chem. Lett. 2011, 2, 768 .

(b) Broccatelli, F.; Mannhold, R.; Moriconi, A.; Giuli, S.; Carosati, E. Mol. Pharm. 2012, 9, 2290.

[3] (a) Tadross, P. M.; Stoltz, B. M. Chem. Rev. 2012, 112, 3550. (b) Bhunia, A.; Yetra, S. R.; Biju, A. T. Chem. Soc. Rev. 2012, 41, 3140 .

(c) Dubrovskiy, A. V.; Markina, N. A.; Larock, R. C. Org. Biomol. Chem. 2013, 11, 191 .

[4] (a) Pialat, A.; Liegault, B.; Taillefer, M. Org. Lett. 2013, 15, 1764. (b) Yang, Z.-W.; Zhang, Q.; Jiang, Y.-Y.; Li, L.; Xiao, B.; Fu, Y. Chem. Commun. 2016, 52, 6709.

(c) Nakazawa, H.; Sako, M.; Masui, Y.; Kurosaki, R.; Yamamoto, S.; Kamei, T.; Shimada, T. Org. Lett. 2019, 21, 6466.

[5] Norihiko, Y.; Tsuyoshi, F.; Takao, M.; Akira, S. Chem. Lett. 1991, 20,459 .

[6] (a) Stang, P. J.; Hanack, M.; Subramanian, L. R. Synthesis 1982, 85. (b) Ritter, K. Synthesis 1993, 735.

[7] Seganish, W. M.; DeShong, P. J. Org. Chem. 2004, 69, 1137. 
[8] (a) Qing, F.-L.; Fan, J.; Sun, H.-B.; Yue, X.-J. J. Chem. Soc., Perkin Trans. 1 1997, 3053.

(b) Frantz, D. E.; Weaver, D. G.; Carey, J. P.; Kress, M. H.; Dolling, U. H. Org. Lett. 2002, 4, 4717.

(c) Gill, D.; Hester, A. J.; Lloyd-Jones, G. C. Org. Biomol. Chem. 2004, 2, 2547.

[9] Effenberger, F.; Mack, K. E. Tetrahedron Lett. 1970, 11, 3947.

[10] (a) Hendrickson, J. B.; Bergeron, R. Tetrahedron Lett. 1973, 14, 4607.

(b) Bengtson, A.; Hallberg, A.; Larhed, M. Org. Lett. 2002, 4, 1231

[11] Zhu, J.; Bigot, A.; Elise, M.; Dau, T. H. Tetrahedron Lett. 1997, 38, 1181.

[12] Wentworth, A. D.; Wentworth, P.; Mansoor, U. F.; Janda, K. D. Org. Lett. 2000, 2, 477.

(b) Chung, C. W. Y.; Toy, P. H. Tetrahedron 2005, 61, 709

[13] (a) White, K. L.; Mewald, M.; Movassaghi, M. J. Org. Chem. 2015, 80,7403 . (b) Yogendra, S.; Hennersdorf, F.; Bauzá, A.; Frontera, A.; Fischer, R.; Weigand, J. J. Chem. Commun. 2017, 53, 2954.

[14] Wu, J.; Lu, C.; Lu, L.; Shen, Q. Chin. J. Chem. 2018, 36, 1031.

[15] Takeshi, K.; Hideki, K.; Takeshi, F.; Rieko, T.; Takashi, T.; Koji, S.; Naokid, T. US 2011/112103, 2011.

[16] Ma, X.; Dang, H.; Rose, J. A.; Rablen, P.; Herzon, S. B. J. Am. Chem. Soc. 2017, 139, 5998.

[17] James M. B.; Daniel C. B.; Timothy, B.; Allen, B. WO 2017/218922, 2017.

[18] Qin, L.; Ren, X.; Lu, Y.; Li, Y.; Zhou, J. Angew. Chem., Int. Ed. 2012, 51, 5915 .

[19] Scheidt, F.; Neufeld, J.; Schäfer, M.; Thiehoff, C.; Gilmour, R. Org. Lett. 2018, 20, 8073.

[20] Chao, J.; Istvan J. E.; Kevin, G.; Richard, H. WO 2014/8214, 2014.

[21] Su, N.; Theorell, J. A.; Wink, D. J.; Driver, T. Angew. Chem., Int. Ed. 2015, 54, 12942.

[22] Picado, A; Li, S.; Dieter, R. K. J. Org. Chem. 2016, 81, 1391.

(Cheng, F.) 